\title{
Narrow band imaging in gynecology: A new diagnostic approach with improved visual identification (Review)
}

\author{
IORI KISU, KOUJI BANNO, KOSUKE TSUJI, KENTA MASUDA, ARISA UEKI, YUSUKE KOBAYASHI, \\ WATARU YAMAGAMI, NOBUYUKI SUSUMU and DAISUKE AOKI
}

Department of Obstetrics and Gynecology, School of Medicine, Keio University, Tokyo, Japan

Received July 27, 2011; Accepted September 9, 2011

DOI: $10.3892 /$ ijo.2011.1226

\begin{abstract}
Narrow band imaging (NBI) is a new endoscopic technique in which images of mucosal microstructures and capillary structures are enhanced by shifting the light spectrum to a narrow band. Image-enhanced gastrointestinal endoscopy using NBI has improved the qualitative diagnosis of the grade and depth of invasion of an atypical lesion. NBI is currently not commonly used in gynecological endoscopy, but has recently been applied in laparoscopy and hysteroscopy. The utility of NBI for diagnosis of endometrial lesions and endometriosis has also been shown. In gynecological endoscopy, NBI provides enhanced images of mucosal microstructures and capillary structures and improves visual identification of lesions. Therefore, image-enhanced observation using NBI is likely to be useful for improved detection of lesions in endoscopic diagnosis. However, this technique remains experimental so far, and no study has demonstrated improved clinical outcome using this technique. In this review, we discuss the utility and potential applications of NBI in clinical practice in gynecology.
\end{abstract}

\section{Contents}

1. Introduction

2. Technical background of narrow band imaging

3. Appearance of NBI-enhanced images

4. Use of NBI in gynecology

5. Conclusion

\section{Introduction}

Endoscopic diagnosis has markedly improved with advances in endoscopic technology, including the greater magnification of

Correspondence to: Dr Kouji Banno, Department of Obstetrics and Gynecology, School of Medicine, Keio University, 35 Shinanomachi, Shinjuku-ku, Tokyo 160-8582, Japan

E-mail:kbanno@sc.itc.keio.ac.jp

Key words: narrow band imaging, endometriosis, endometrial lesions, hysteroscopy, laparoscopy, colposcopy the high-pixel endoscope, and new endoscopic examinations are emerging that complement conventional observation. Among these techniques, narrow band imaging (NBI) for imageenhanced endoscopy has dramatically changed diagnostic procedures for some organs, especially in the gastrointestinal system. NBI is a new technique for enhancement of images of mucosal microstructures and capillary structures that is achieved by shifting the light spectrum to a narrow band. NBI is widely used in clinical practice for the gastrointestinal system, including the pharynx, esophagus, stomach and large intestine, and for regions such as the oro-pharynx, lung and urinary tract. This method has produced a remarkable improvement in qualitative diagnosis of the grade and depth of invasion of atypical lesions, and permits earlier lesion detection (1-4). The NBI method is also referred to as 'optical biopsy', a visual diagnosis that needs no biopsy, and diagnostic criteria for various organs are being developed based on pattern classification of mucosal microvessels and microstructures $(5,6)$.

Studies using NBI for gynecologic endoscopy have shown the utility of the approach, but NBI is still not commonly used in the gynecological field. In this review, we focus on the use of NBI in clinical practice in gynecology and discuss the potential role of the NBI technique as a new method for endoscopic diagnosis in the gynecological field.

\section{Technical background of narrow band imaging}

NBI was developed by the Japanese National Cancer Center Hospital and Olympus Medical Systems (Tokyo, Japan) in 1999. Sano et al (Gastrointest Endosc 53: abs. 125, 2001) first described the clinical utility of NBI for gastrointestinal endoscopy in 2001. The new characteristics of NBI are the narrow band spectrum of the light used in the endoscope, which increases lesion visibility and improves the observation of superficial structures and microvessels due to the scattering characteristics of the light; i.e., light of short and long wavelengths provides superficial and deep layer information, respectively. Studies of combinations of different wavelengths showed that a combination of 415 and $540 \mathrm{~nm}$ provided the highest visibility. Therefore, the current NBI system uses these two wavelengths (7).

The visible light wavelength for human is $400-700 \mathrm{~nm}$, and different wavelengths appear in different colors. Light of $400 \mathrm{~nm}$ appears blue, that of $550 \mathrm{~nm}$ is green, and that of 


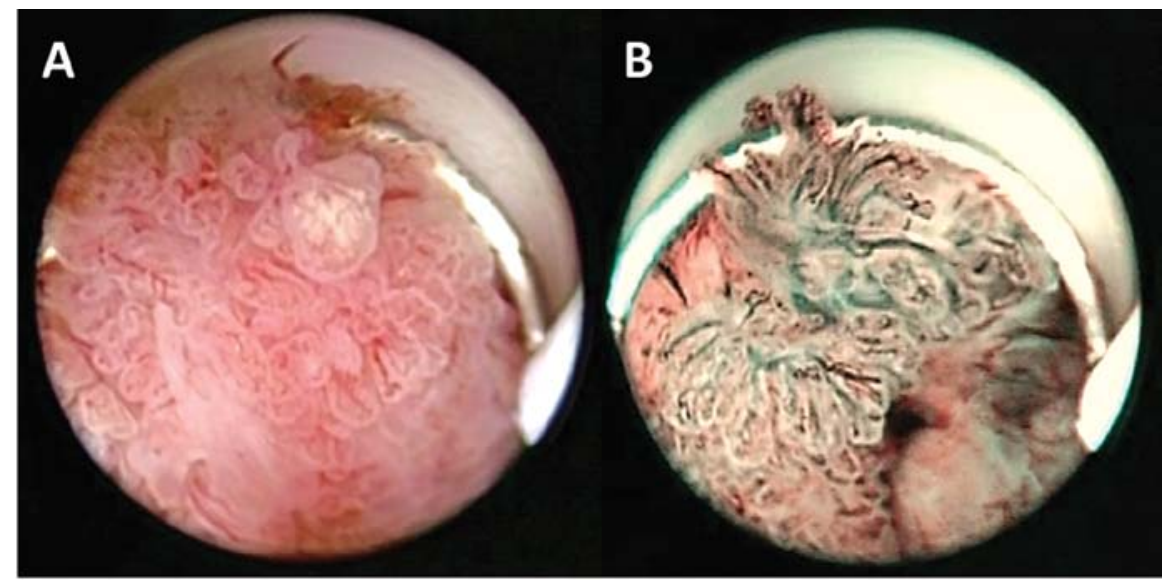

Figure 1. Comparison of visualization of endometrial cancer by (A) white light and (B) NBI hysteroscopy. Papillary protruding lesions can be observed, and expanded, inflectional and coiling atypical vessels are more clearly imaged in dark green on the endometrial surface by NBI.

$600 \mathrm{~nm}$ is red, while light with a wide range of wavelengths of 400-700 nm appears white. In NBI, optical filters positioned in front of a xenon lamp are inserted into the light path, leading to irradiation of the mucosa with two narrow band wavelengths $(415 \pm 30$ and $540 \pm 30 \mathrm{~nm})$. The peak absorbance of oxidized hemoglobin in the blood occurs at 415 and $540 \mathrm{~nm}$ and most of the blue light emitted in the NBI system is absorbed by hemoglobin, resulting in blood vessels appearing as a dark red color. Other tissue scatters the light and appear as a bright color. Therefore, NBI is an imaging technique that displays a clear contrast between blood vessels in the mucosal surface and other tissue.

In this system, there are no special requirements or patient preparation for NBI, and observation with conventional white light and NBI is interchangeable at the touch of a button by the surgeon.

\section{Appearance of NBI-enhanced images}

NBI gives enhanced images of the mucosal microstructure and microvascular structure, and is particularly useful for evaluation of microvessels. Neoplastic vessels develop in the surface of tumor lesions, and thus NBI is very useful for observation of microvessels for qualitative diagnosis of tumors and non-tumor lesions $(1,5,6,8-10)$. In contrast, evaluation of the microvascular structure with conventional white light endoscopy is difficult. Gynecologic studies have also shown angiogenesis in tumor lesions and atypical vessels in malignant lesions $(11,12)$. An NBI-based hysteroscopic image of endometrial cancer is shown in Fig. 1. In this image, vessels on the endometrial surface are displayed in dark blue, in marked contrast with these vessels in white light images. Thus, the microvascular structure, angiogenesis and atypical vessels in malignant lesions are easy to observe using NBI, which facilitates detection of lesions.

\section{Use of NBI in gynecology}

A search of the PubMed database was performed in this review using the keywords 'narrow band imaging', 'hysteroscopy', 'laparoscopy', and 'colposcopy'. Farrugia et al (13) first described the application of NBI in gynecology in 2007.
Subsequently, reports on applications of NBI in gynecology have increased to 16 articles, as summarized in Table I, and currently NBI is often used to identify endometrial lesions by hysteroscopy and peritoneal lesions by laparoscopy.

Endometrial lesions. Hysteroscopy is considered to be the best technique for examination of the uterine cavity $(14,15)$ and many studies comparing hysteroscopy and histology have shown that hysteroscopy has a higher correct diagnostic rate for abnormal lesions in the uterine cavity and endometrial cancer (16-18). However, Lasmar et al (19) found that the sensitivities of hysteroscopy with white light for diagnosis of endometrial hyperplasia and endometrial cancer were $56.3 \%$ and $80.0 \%$, respectively, and suggested that visual identification based on morphological changes in an endometrial lesion were inadequate and could be subjective, indicating the need for pathological examination for diagnosis of endometrial lesions (20). However, hysteroscopic endometrial biopsy occasionally requires use of thick operative sheaths and cervical dilation, resulting in problems including pain and risk of complications. Diagnosis by biopsy may also not always be correct, and it has been shown that $42.6 \%$ of patients diagnosed with atypical endometrial hyperplasia (AEH) by biopsy actually had endometrial cancer (21).

In 1971, Folkman (22) first showed that tumor growth and progression depended on angiogenesis. Since then, angiogenic intensity has played a diagnostic role for many malignant lesions, including endometrial cancer. In 1999, Abulafia et al (23) examined angiogenesis in endometrial hyperplasia and endometrial cancer, and found angiogenesis in complex endometrial hyperplasia were significantly more than in simple endometrial hyperplasia and significantly less than in endometrial cancer. In 2006, Stefansson et al (24) showed that vessels in endometrial cancer were structurally and functionally abnormal and that the structural changes were related to vascular invasion and decreased survival.

The application of NBI in hysteroscopy facilitates visualization of minute neovascular proliferation and atypical vessels for detection and diagnosis of endometrial lesions. In 2009, Surico et al (25) first applied NBI hysteroscopy in identification of endometrial lesions and showed that NBI clearly visual- 


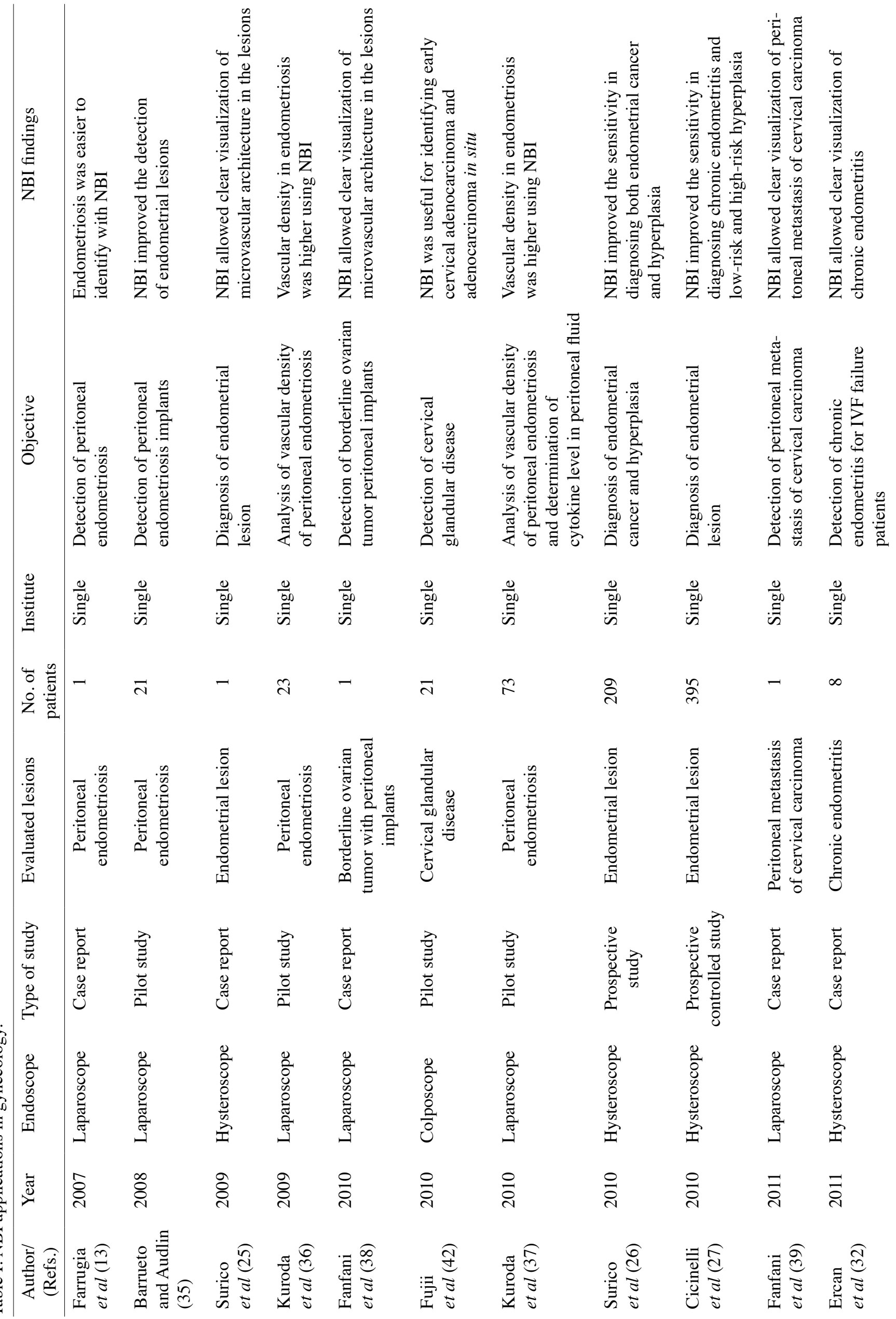




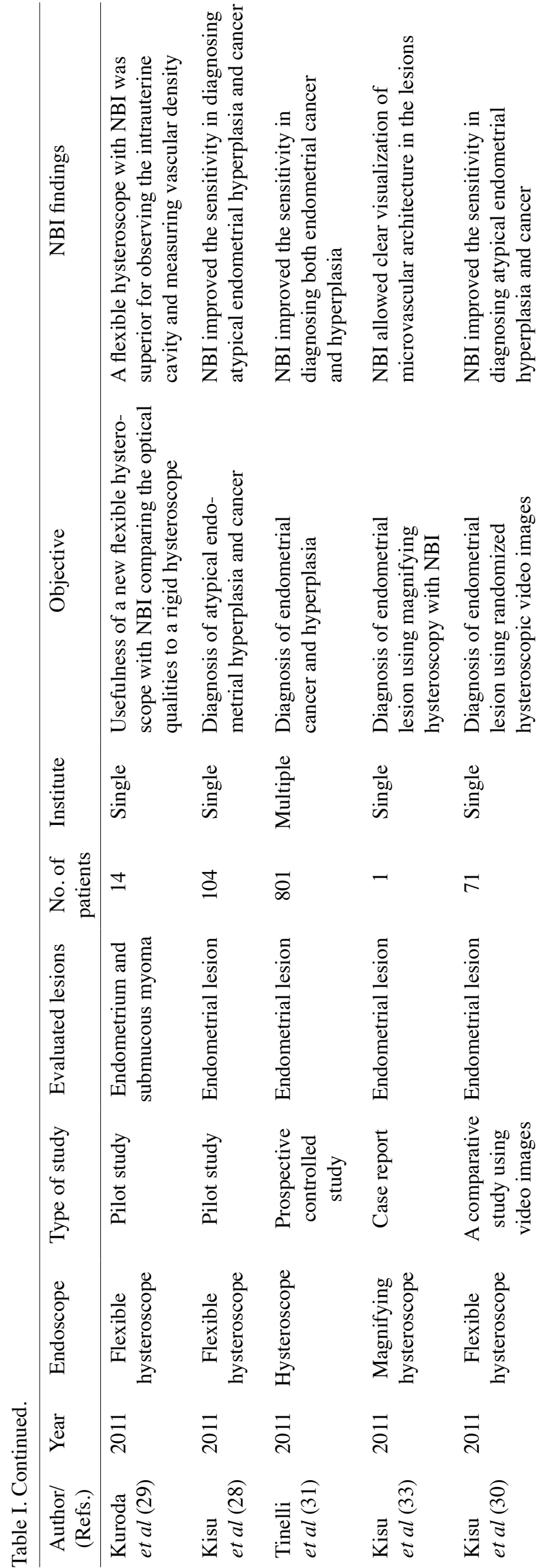

ized the microvascular structure and allowed identification of lesions with irregular microvessels. The improved accuracy of visual identification of endometrial lesions suggested that NBI is a promising methodology for early detection of these lesions. Moreover, Surico et al (26) conducted a prospective comparative study of NBI and white light hysteroscopy for diagnosis of endometrial cancer and hyperplasia in 209 patients with abnormal uterine bleeding. The sensitivity and specificity of white light hysteroscopy were $84.2 \%$ and $99.5 \%$ in endometrial cancer and $64.9 \%$ and $98.8 \%$ in endometrial hyperplasia, whereas these values for NBI hysteroscopy were $94.7 \%$ and $97.9 \%$ in endometrial cancer and $78.4 \%$ and $97.7 \%$ in endometrial hyperplasia, indicating that NBI gave significantly higher sensitivity without loss of specificity for diagnosis of endometrial cancer and hyperplasia. Cicinelli et al (27) also conducted a prospective study in 395 patients to evaluate the utility of NBI hysteroscopy for diagnosis of endometrial lesions. The sensitivity of white light hysteroscopy were $81 \%$ in proliferative endometrium, $70 \%$ in chronic endometritis, $70 \%$ in low-risk hyperplasia and $40 \%$ in high-risk hyperplasia, whereas these values for NBI hysteroscopy were $92 \%, 88 \%, 88 \%$ and $60 \%$, respectively, indicating that NBI hysteroscopy significantly improved the sensitivity for diagnosis of these lesions.

We have also used NBI with a flexible hysteroscope and showed the utility of this method in endoscopic diagnosis of malignant endometrial lesions, in comparison with historical data collected using conventional hysteroscopy (28). NBI hysteroscopy in addition to conventional white light hysteroscopy were conducted in 104 patients who visited our clinic for suspected lesions in the uterine cavity. The accuracy for diagnosis of endometrial lesions in these patients were $88.5 \%$ in NBI hysteroscopy and $70.8 \%$ in conventional hysteroscopy which was conducted in 209 patients previously, indicating that NBI hysteroscopy gave significantly higher accuracy for diagnosis of endometrial lesions. The sensitivities for diagnosis of AEH or carcinoma were $97.2 \%$ and $82.6 \%$ for NBI hysteroscopy and conventional hysteroscopy, respectively, and the sensitivities using NBI hysteroscopy were significantly higher than those of the conventional method with no loss of specificity. Kuroda et al (29) also used NBI with flexible hysteroscopy and suggested that this approach was superior to rigid hysteroscopy for observation of the uterine cavity and measurement of vascular density using vascular analysis software.

We have recently reported the utility of NBI hysteroscopy for diagnosis of malignant endometrial lesions by comparison of the sensitivity and specificity between white light alone and white light + NBI using randomized video images (30). This is the first report of comparative study compared two methods using randomized video images. Video images from 65 patients were edited into two groups, white light alone (WL group) and white light + NBI (NBI group) (130 images in total). Computerized block randomization of the order was then performed, and four raters independently diagnosed the images without use of other clinical information. The sensitivity of diagnosis of AEH or endometrial carcinoma was numerically higher in the NBI group for all raters, and the average sensitivity was significantly higher in the NBI group compared to the WL group (78.6\% vs. $63.7 \%$ ). The specificity for each rater and the average specificity were comparable between the two groups. 
Tinelli et al (31) also conducted the first multicenter study of NBI in gynecology in 801 outpatients. The sensitivities of NBI hysteroscopy and white light hysteroscopy were $93 \%$ and $81 \%$ for endometrial cancer, $82 \%$ and $56 \%$ for low-risk hyperplasia, and $60 \%$ and $20 \%$ for high-risk hyperplasia, indicating significantly higher sensitivity of NBI hysteroscopy. This suggested that NBI hysteroscopy could be useful for reducing the risk of missing severe pathologies and improving the diagnosis of preneoplastic and neoplastic pathologies, with the conclusion that NBI hysteroscopy had very high diagnostic accuracy for the exploration of the uterine cavity. This study is the largest clinical trial performed in this area and provided objective evidence for the utility of NBI for detection of gynecological tumors in clinical practice.

Ercan et al (32) have suggested that NBI may also be useful to determine the cause of infertility. NBI hysteroscopy was conducted to find abnormalities in the uterine cavity in patients with previous failed in vitro fertilization with assisted reproductive techniques. Endometritis was found in 3 of 8 patients by NBI hysteroscopy, but not by white light hysteroscopy. Endometritis may be a cause of implantation failure, which will reduce the chance of pregnancy in in vitro fertilization. Therefore, NBI may be useful for evaluation of causes of infertility that involve the endometrium. We have also applied magnifying hysteroscopy with NBI for endometrial lesions (33). Magnifying hysteroscopy with NBI enabled fine visualization of the microstructure and blood microvessels in an endometrial lesion. This preliminary experience indicates that it is likely to have a role in 'optical biopsy' in the future, followed on the gastrointestinal field.

All studies on NBI hysteroscopy have shown its utility for detection and diagnosis of endometrial lesions, since NBI facilitates visualization of microvascular structures and microstructures on the endometrial surface. These results indicate that diagnostic criteria for endometrial lesions are required based on pattern classification of mucosal microvessels and microstructures visualized by NBI, as already established in gastrointestinal endoscopy.

Endometriosis. Endometriosis is defined as the presence of endometrial glands and stroma outside of the uterine cavity. This condition often develops in women of reproductive age and some patients have severe pain and are often treated by surgery. In patients with peritoneal endometriosis, laparoscopic resection extends the pain-free interval; however, microlesions cannot be distinguished from normal tissues and not all endometriosis lesions are macroscopically identified, resulting in incomplete relief of pain after surgery. Macroscopic identification of endometriosis lesions is currently poor, with histological examination having a positive predictive value of $65 \%$ for suspect lesions in laparoscopy (34). NBI may facilitate macroscopic identification of peritoneal endometriosis through imaging of microvascular structures, since inflammatory reactions, angiogenesis and changes in the microvascular structure occur in the early stage of lesion formation.

Farrugia et al (13) first showed that microvascular imaging using NBI permitted more definite identification of endometriosis lesions, in comparison with imaging by white light, and suggested that NBI could be used for diagnosis of endometriosis lesions. Barrueto and Audlin (35) conducted pelviscopy in 20 patients with pelvic pain and possible pelvic endometriosis before surgery, initially using white light and then NBI. Fourteen patients had lesions that were not detected by white light, but were detected by NBI and were subsequently definitively diagnosed as endometriosis by biopsy. Thus, Barrueto and Audlin concluded that NBI was effective for identifying endometriosis implants that were invisible under white light. Kuroda et al $(36,37)$ assumed that angiogenesis is needed to develop or maintain peritoneal endometriosis and that the activity of endometriosis is related to vascularity. In an analysis of the vascular density using NBI and vascular analysis software, three colors (red, black and white) were used to display lesions of peritoneal endometriosis. Red lesions were considered to be the most active, with NBI providing clear images of lesions and surrounding vessels. The median difference in vascular density between NBI and the conventional method was significantly greater for red lesions with angiogenesis than for black and white lesions. Therefore, Kuroda et al concluded that red lesions were indicative of early-stage endometriosis with angiogenesis and that NBI was superior for detection of capillary blood vessels with significantly higher peritoneal vascular density, compared to the conventional method.

These studies have shown the utility of NBI for detection of peritoneal endometriosis, since NBI facilitates visualization of microvascular structures on the peritoneum. Excision of the maximal number of endometriotic lesions provides the patient with the opportunity for delayed recurrence or treatment of the disease. Although there is no study to assess whether the clinical outcomes are improved, NBI may be a useful technique for increasing the detection of endometriosis and improving patient clinical outcomes.

Peritoneal metastasis. The presence of peritoneal implants in gynecological cancer is an important factor for determining the disease stage and guiding intraoperative changes in surgical procedures and postoperative adjuvant therapy. Intraoperative detection of peritoneal implants and histologic confirmation depend on the surgeon's experience and the structural characteristics of the implant lesion. Diagnosis of implants is conducted by endoscopic biopsy and improved visualization of lesions facilitates their identification. Fanfani et al $(38,39)$ applied NBI for visualization of the microvascular architecture in suspected peritoneal implant lesions in cases of borderline ovarian tumor and cervical carcinoma. NBI increased the contrast of the microvasculature and inflammatory response in comparison with normal peritoneum, leading to clear observation of lesions. Thus, Fanfani et al suggested that NBI was a new tool for detection of peritoneal implants in gynecological cancer and a useful method to assist intraoperative determination of surgical procedures, disease progress, and the need for postoperative treatment.

Cervical lesions. Colposcopy has been used to observe cervical lesions since 1925 (40). Colposcopic criteria for cervical dysplasia and squamous cell carcinoma are established, but those for cervical glandular disease and adenocarcinoma are unknown. Wright (41) established disease features based on surface and vessel patterns; however, small abnormal lesions and minute changes are unlikely to be detected by colposcopy. Therefore, Fujii et al (42) evaluated the utility of NBI colposcopy in diagnosis of adenocarcinoma in situ (AIS) and adenocarcinoma. NBI 
colposcopy provided clearer images of microvascular structures in the cervical surface compared to conventional colposcopy, with waste thread-like, dot-like and root-like vascular patterns found in 18 of $21(86 \%)$ patients with AIS and adenocarcinoma. These results show that NBI colposcopy is useful for detection of AIS and early adenocarcinoma.

The vascular structure in glandular disease is not specific and is also observed in immature metaplastic epithelium, condyloma and squamous cell carcinoma. Furthermore, half of cases of AIS develop simultaneously with cervical intraepithelial neoplasia and histology is needed for definitive diagnosis. Optoelectronic devices, Raman spectra device and dynamic spectral imaging have recently been developed, but these techniques are applied only to cervical intraepithelial neoplasia detection, and not for glandular disease. Therefore, NBI may be a useful technique for increasing the sensitivity of diagnosis of glandular disease. However, this study is an exploratory report, and has not demonstrated improved clinical outcome. Further study is required to prove the usefulness in this field.

\section{Conclusion}

NBI is a method for enhancement of endoscopic images that has attracted attention, especially in the gastrointestinal field. Image-enhancement endoscopy by NBI has markedly changed endoscopic diagnosis. In gynecological endoscopy, NBI has mainly been used in diagnosis of endometrial lesions and endometriosis and its utility is drawing interest. Application of NBI to intraperitoneal examination of peritoneal lesions including peritoneal endometriosis and implants is likely to improve evaluation and detection of lesions and provide accurate information on the disease stage. In turn, this should lead to improved treatment. In diagnosis of endometrial lesions, cytology and histology often give false-negative results for localized lesions and the tests also cause pain. Targeted biopsy using a rigid hysteroscope can give a more accurate diagnosis, but this method often requires use of a cervical dilatation. This causes pain, has associated risks, and is difficult to perform in many clinics. In contrast, if NBI hysteroscopy improves visual diagnosis, this approach will allow less-invasive diagnosis with a flexible hysteroscope, reduce unnecessary cytological and histological tests, and the associated pain, and be likely to be efficient for early detection of lesions.

In this review, we focused on the application of NBI in clinical practice in gynecology. In gynecological endoscopy, NBI provides enhanced images of mucosal microstructures and capillary structures, which improves visual identification of lesions. The excellent detection of lesions suggests that this method has great potential as a new approach for 'optical biopsy' in diagnostic gynecological endoscopy, similarly to its current role in gastrointestinal endoscopy. However, further large-scale multicenter randomized trials are required to confirm the value of NBI in gynecological applications.

\section{Acknowledgments}

We thank Dr Tai Ohmori (Department of Surgery, School of Medicine, Keio University), and Takashi Saga, Sayoko Tanaka, Ayumi Saito and Kenji Matsushima (Olympus Medical Science Sales Co., Ltd) for their advice on the NBI system, and
Shigeru Owa (AVS Co., Ltd) for his technical assistance. This study was supported by the Graduate School Doctoral Student Grant-in-Aid Program 2011, a grant from Training for Oncology Professionals in 9 universities (Integrative Research Program 2011), Keio University Grant-in-Aid for Encouragement of Young Medical Scientists and JFE (The Japanese Foundation for Research and Promotion of Endoscopy) Grant.

\section{References}

1. Machida H, Sano Y, Hamamoto Y, Muto M, Kozu T, Tajiri H and Yoshida S: Narrow-band imaging in the diagnosis of colorectal mucosal lesions: a pilot study. Endoscopy 36: 1094-1098, 2004.

2. Irjala H, Matar N, Remacle M and Georqes L: Pharyngo-laryngeal examination with the narrow band imaging technology: early experience. Eur Arch Otorhinolaryngol 268: 801-806, 2011.

3. Zaric B and Perin B: Use of narrow-band imaging bronchoscopy in detection of lung cancer. Expert Rev Med Devices 7: 396-406, 2010.

4. TraxerO,Geavlete B,De Medina SG,Sibony Mand Al-Qahtani SM: Narrow-band imaging digital flexible ureteroscopy in detection of upper urinary tract transitional-cell carcinoma: initial experience. J Endourol 25: 19-23, 2011.

5. Inoue $\mathrm{H}$, Honda $\mathrm{T}$, Nagami $\mathrm{K}$, et al: Ultra-high magnification endoscopic observation of carcinoma in situ of the oesophagus. Dig Endosc 9: 16-18, 1997.

6. Yao K and Oishi T: Microgastroscopic findings of mucosal microvascular architecture as visualized by magnifying endoscopy. Dig Endosc 13: S27-S33, 2001.

7. Gono K, Obi T, Yamaguchi M, et al: Appearance of enhanced tissue features in narrow-band endoscopic imaging. J Biomed Opt 9: 568-577, 2004.

8. Muto $\mathrm{M}$ and Horimatsu T: Narrow-band imaging of the gastrointestinal tract. J Gastroenterol 44: 13-25, 2009.

9. Muto M, Nakae M, Katada C, et al: Squamous cell carcinoma in situ at oropharyngeal and hypopharyngeal mucosal sites. Cancer 101: 1375-1381, 2004.

10. Shibuya K, Hoshino H, Chiyo M, et al: High magnification bronchovideoscopy combined with narrow band imaging could detect capillary loops of angiogenic squamous dysplasia in heavy smokers at high risk for lung cancer. Thorax 58: 989-995, 2003.

11. Gazvani R and Templeton A: Peritoneal environment, cytokines and angiogenesis in the pathophysiology of endometriosis. Reproduction 2132: 217-226, 2002.

12. Groothius PG, Nap AW, Winterhager E and Grummer R: Vascular development in endometriosis. Angiogenesis 8: 147-156, 2005.

13. Farrugia M, Nair $M$ and Kontronis K: Narrow band imaging in endometriosis. J Minim Invasive Gynecol 14: 393-394, 2007.

14. Balasch J: Investigation of the infertile couples: investigation of the infertile couple in the era of assisted reproductive technology: a time for reappraisal. Hum Reprod 15: 2251-2257, 2000.

15. Brand A: Diagnosis of endometrial cancer in women with abnormal uterine bleeding. J SOGC 86: 1-3, 2000.

16. Chanbers JT and Chanbers SK: Endometrial sampling: when? where? why? with what? Clin Obstet Gynecol 35: 28-39, 1992.

17. Creasman WT: Endometrial cancer: incidence, prognostic factors, diagnosis, and treatment. Semin Oncol 24 (Suppl 1): 140-150, 1997.

18. Garuti G, Sambruni I, Colonnelli M and Luerti M: Accuracy of hysteroscopy in predicting histopathology of endometrium in 1500 women. J Am Assoc Gynecol Laparosc 8: 207-213, 2001.

19. Lasmar RB, Barrozo PR, De Oliveira MA, Coutinho ES and Dias R: Validation of hysteroscopic view in cases of endometrial hyperplasia and cancer in patients with abnormal uterine bleeding. J Minim Invasive Gynecol 13: 409-412, 2006.

20. Zola FE, Nogueira AA, De Andrade JM and Candido dos Reis FJ: Hysteroscopic appearance of malignant and benign endometrial lesions: a case-control study. Arch Gynecol Obstet 275: 49-52, 2007.

21. Trimble CL, Kauderer J, Zaino R, et al: Concurrent endometrial carcinoma in women with a biopsy diagnosis of atypical endometrial hyperplasia: a Gynecologic Oncology Group study. Cancer 106: 812-819, 2006.

22. Folkman J: Tumor angiogenesis. N Engl J Med 285: 1182-1186, 1971. 
23. Abulafia O, Triest WE and Sherer DM: Angiogenesis in malignancies of the female genital tract. Gynecol Oncol 72: 220-231, 1999.

24. Stefansson IM, Salvesen HB and Akslen LA: Vascular proliferation is important for clinical progress of endometrial cancer Cancer Res 66: 3303-3309, 2006.

25. Surico D, Viqone A and Leo L: Narrow band imaging in endometrial lesions. J Minim Invasive Gynecol 16: 9-10, 2009.

26. Surico D, Vigone A, Bonvini D, Tinelli R, Leo L and Surico N Narrow-band imaging in diagnosis of endometrial cancer and hyperplasia: a new option? J Minim Invasive Gynecol 17: 620-625, 2010.

27. Cicinelli E, Tinelli R, Colafiqlio G, Pastore A, Mastrolia S, Lepera A and Clevin L: Reliability of narrow-band imaging (NBI) hysteroscopy: a comparative study. Fertil Steril 94: 2303-2307, 2010.

28. Kisu I, Banno K, Kobayashi Y, et al: Flexible hysteroscopy with narrow band imaging (NBI) for endoscopic diagnosis of malignant endometrial lesions. Int J Oncol 38: 613-618, 2011.

29. Kuroda K, Kitade M, Kikuchi I, et al: A new instrument: a flexible hysteroscope with narrow band imaging system - optical quality comparison between a flexible and a rigid hysteroscope. Minim Invasive Ther Allied Technol doi:10.3109/13645706.2010. 548935, 2011.

30. Kisu I, Banno K, Kobayashi Y, et al: Narrow band imaging hysteroscopy: a comparative study using randomized video images. Int J Oncol 39: 1057-1062, 2011.

31. Tinelli R, Surico D, Leo L, et al: Accuracy and efficacy of narrowband imaging versus white light hysteroscopy for the diagnosis of endometrial cancer and hyperplasia: a multicenter controlled study. Menopause 18: 1026-1029, 2011.

32. Ercan CM, Ozturk M, Dede M, Coksuer H, Karasahin KE and Yenen MC: Narrow band imaging hysteroscopy: a new diagnostic technique in recurrent IVF failure? Arch Gynecol Obstet 283 (Suppl 1): 135-136, 2011.
33. Kisu I, Banno K, Susumu N and Aoki D: Magnifying hysteroscopy with narrow-band imaging for visualization of endometrial lesions. Int J Gynaecol Obstet 115: 74-75, 2011.

34. Marchino G, Gennarelli G, Enria R, et al: Diagnosis of pelvic endometriosis with use of macroscopic vs histologic findings. Fertil Steril 84: 12-15, 2005.

35. Barrueto FF and Audlin KM: The use of narrowband imaging for identification of endometriosis. J Minim Invasive Gynecol 15: 636-639, 2008

36. Kuroda K, Kitade M, Kikuchi I, et al: Vascular density of peritoneal endometriosis using narrow-band imaging system and vascular analysis software. J Minim Invasive Gynecol 16: 618-621, 2009.

37. Kuroda K, Kitade M, Kikuchi I, Kumakiri J, Matsuoka S, Kuroda M and Takeda S: Peritoneal vascular density assessment using narrow-band imaging and vascular analysis software, and cytokine analysis in women with and without endometriosis. J Minim Invasive Gynecol 17: 21-25, 2010.

38. Fanfani F, Gallotta V, Rossitto C, Fagotti A and Scambia G: Narrow band imaging in borderline ovarian tumor. J Minim Invasive Gynecol 17: 146-147, 2010.

39. Fanfani F, Rossitto C, Fagotti A, Gallotta V, Gagliardi ML and Scambia G: Narrow-band imaging in laparoscopic management of cervical carcinoma. J Minim Invasive Gynecol 18: 146-147, 2011.

40. Jordan JA and Singer A: Colposcopy. In: The Cerix. Jordan JA and Singer A (eds). Blackwell Publishing, Malden, MA, pp445-461, 2006.

41. Wright VC: Colposcopy of adenocarcinoma in situ and adenocarcinoma of the uterine cervix: differentiation from other cervical lesions. J Low Genit Tract Dis 3: 83-97, 1999.

42. Fujii T, Nakamura M, Kameyama K, et al: Digital colposcopy for the diagnosis of cervical adenocarcinoma using a narrow band imaging system. Int J Gynecol Cancer 20: 605-610, 2010. 